

\title{
New Method Based on Priority of Heterogeneous Traffic for Scheduling Techniques in M2M Communications over LTE Networks
}

\author{
Mariyam Ouaissa $^{1 *} \quad$ Abdallah Rhattoy $^{2}$ \\ ${ }^{1}$ Information and Communication Systems Engineering Research Group, High School of Technology, \\ Mathematical Modeling and Computer Science Laboratory, Ecole Nationale Supérieure des Arts et Métiers, \\ Moulay-Ismail University, Meknes, Morocco \\ ${ }^{2}$ Department of Computer, Information and Communication Systems Engineering Research Group, \\ High School of Technology, Moulay-Ismail University, Meknes, Morocco \\ * Corresponding author’s Email: mariyam.ouaissa@edu.umi.ac.ma
}

\begin{abstract}
Machine to Machine (M2M) communication is a technology for machines communicating with each other without human intervention. Long Term Evolution (LTE) and Long-Term Evolution-Advanced (LTE-A) cellular networks technologies provide the resources required for M2M communication. However, LTE is required to meet M2M communication requirements, such as power management and support for a large number of devices while maintaining the Quality of Service (QoS) requirements for these devices. Resource allocation or scheduling is one of the key challenges facing M2M communications over Long-Term Evolution networks. M2M traffic has unique characteristics; it usually consists of a large number of small data packets, with specific delays, generated by a potentially large number of devices competing on scarce radio resources. In this article, we will present the scheduling techniques on the Packet Downlink Control Channel (PDCCH) in a M2M scenario based on the priority and differentiation between heterogeneous traffic (real time and non-real time) to achieve high spectral efficiency in cellular systems and provide quality of service guarantees for system users. The results obtained show that the FLS downlink scheduler is the optimal solution based on our proposed method which offers more priority to the real time service (video and voip) than the non real time service (best effort).
\end{abstract}

Keywords: M2M, MTC, H2H, LTE, LTE-A, Scheduling, QoS, Resource allocation.

\section{Introduction}

Machine to Machine (M2M) communication [1] is a new paradigm that allows a myriad of machines to communicate with each other autonomously. M2M thus allows the emergence of new services belonging to different fields of application such as transport, health and surveillance thus improving the daily life of human. M2M has been standardized in Long Term Evolution (LTE) cellular networks by Machine Type Communications (MTC) [2, 3].

The design of the LTE cellular system is used for Human to Human (H2H) communication. Therefore, it is considered that the M2M application platform is inappropriate. Its obvious features include for example, the number of devices in M2M communications is greater than that of $\mathrm{H} 2 \mathrm{H}$ communications. It also contains a small amount of data and a large number of nodes, different delays and diversified services which can cause a sharp increase or decrease in traffic [4]. Therefore, as a M2M communication network, many problems are encountered by the mobile communication network designed based on the characteristics of $\mathrm{H} 2 \mathrm{H}$. In order to realize that it can efficiently provide a M2M communications service, the existing mobile communication network must be optimized based on the M2M communication applications to avoid congestion or network overhead caused by simultaneous access of huge amounts of M2M users to the network $[5,6]$.

As a result, many standards organizations and 


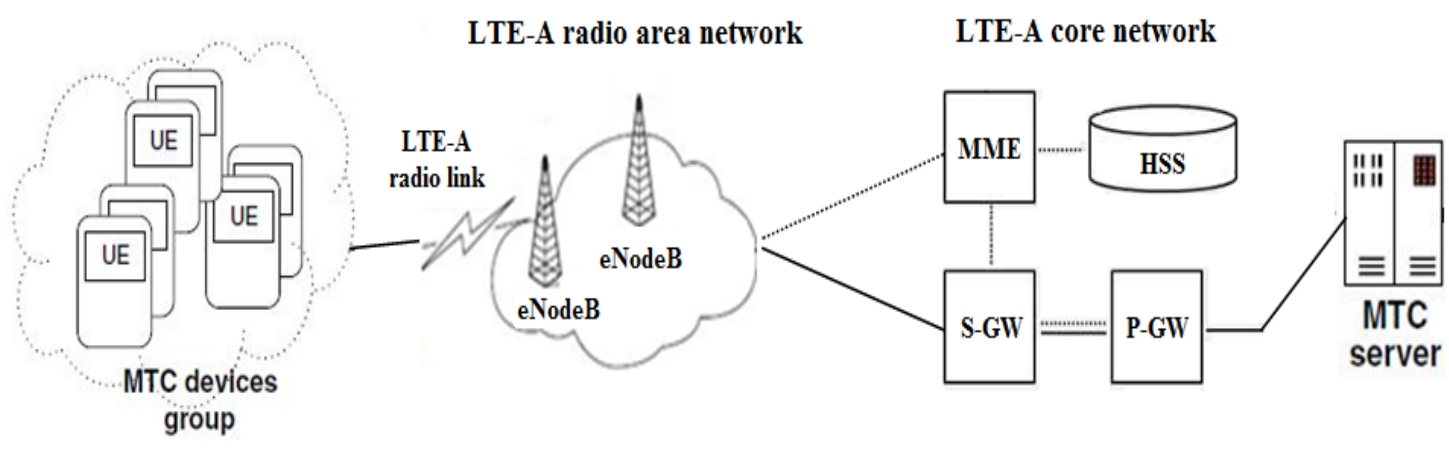

Figure.1 M2M system architecture

international projects are making great efforts to improve the support of LTE cellular networks for M2M applications. A key feature of the LTE network is the adoption of a number of advanced radio resource management procedures to improve system performance and support user needs for Quality of Service (QoS), especially for real time flows to know voice and video. In particular, packet scheduling mechanisms play a fundamental role because they are responsible for choosing, carefully and in a precise time, how to distribute the radio resources between the different stations taking into account the channel conditions and requirements of QoS [7].

In this context, we are interested in the issues of radio resource allocation and its impact on QoS within a LTE candidate technology for M2M communication. The purpose of our work is to create a M2M scenario over LTE downlink network by adding a service priority method between realtime and non-real time traffics in order to simulate the main downlink scheduling techniques of which the goal is to find the best scheduler that offer an acceptable QoS level according to a priority and a differentiation between services, where real-time service is certainly prior to the non-real-time service and the allocation of resources for the service in real time is prioritized at non real time. Moreover, the proposed scheduler aimed in particular at improving the performance metrics for real time services and maintaining in general a satisfactory level of the performance metrics for the other services in the network simultaneously. The rest of the paper is organized as follows: in section 2, we presented the M2M system architecture, as well as access techniques for LTE technology focusing on the scheduling technique. We will also explain the resource allocation for M2M communications. The third section deals with the procedure and the policies of the scheduling for the downlink direction as well as the study of the different algorithms used by the LTE technology. In the fourth section, we discussed our method based on service priority. We describe in section 5 the evaluation of scheduling algorithms performance with the method proposed, we analyze the impact of each algorithm on the QoS for each type of real-time traffic (voip and video) and non real-time (best effort) and we compare the results of the simulations by indicating the best algorithm in terms of the following metrics: Throughput, Goodput, Fairness, Delay, Packet Loss Rate (PLR) and Spectral Efficiency.

\section{System model}

\subsection{M2M system architecture}

Fig. 1 shows the architecture of an M2M system, which has three main parts: the MTC devices, the communication network and the MTC server [8].

MTC devices are terminals that provide information, often in real time, devices involved in M2M or MTC communications are generally referred to as Machine Type Communications Devices (MTCD). Unlike H2H communications, M2M communications are typically characterized by massive access combined with small payloads of data. MTCD data can be generated by event triggering or as periodic reports. For the communication network, there are several technologies that can be considered as needed. LTE is the latest evolution of mobile phone standards defined by the 3rd Generation Partnership Project (3GPP). The network consists of two parts: the radio access network Evolved UMTS Terrestrial Radio Access Network (EUTRAN) and the core network Evolved Packet Core (EPC). The core network EPC uses all-IP technology [9]. The M2M server manages visualization, data analysis and the resulting decision-making.

With the rapid development of the Long Term Evolution of cellular networks and its evolution Long Term Evolution-Advanced (LTE-A), M2M communications over extended coverage LTEAdvanced cellular networks should be an important 
part of Internet of Things (IoT). LTE networks are designed to support packet switching with seamless mobility, QoS provisioning and minimal latency. LTE transmissions are organized into radio frames of $10 \mathrm{~ms}$, each frame divided into ten subframes of 1 $\mathrm{ms}$. The subframes are divided into two slots of 0.5 $\mathrm{ms}$. The transmissions are multiplexed using Single Carrier Frequency Division Multiple Access (SCFDMA) in the Uplink (UL) channel, while Orthogonal Frequency Division Multiple Access (OFDMA) is used in the Downlink (DL) channel. Scheduling is the process implemented by Evolved NodeB (eNodeB) to allocate radio resources based on human User Equipment (UE) or MTCD requests in the downlink or uplink direction. The minimum radio resource unit that can be allocated to a device is called the Physical Resource Block (PRB), which is composed of two slots in the time domain and 12 subscribers that correspond to $180 \mathrm{kHz}$ in the frequency domain $[10,11]$.

\subsection{Downlink Scheduling Process}

Scheduler is the functional entity for obtaining fast packet scheduling and the basic MAC layer function in LTE eNodeB. This has a major impact on system performance. It contains an uplink scheduler and a downlink scheduler, which support the allocation of physical layer resources for Uplink Shared Channel (UL-SCH) and Downlink Shared Channel (DL-SCH) respectively. Physical layer resources include primarily PRB, Modulation Coding Scheme (MCS), power allocation schemes, and antenna selection schemes for multiple antennas. Fig. 2 shows the position of the scheduler in the LTE protocol and its relation to key techniques such as Hybrid Automatic Repeat Request (HARQ) and Adaptive Modulation and Coding (AMC). Its key function is to determine how to properly allocate the resource to users in order to maximize system throughput, as long as the time and packet QoS are guaranteed $[12,13]$.

The quality of the channel is necessary in the scheduling. The eNodeB gets it by receiving the notification of the user's channel quality indicator. As shown in Fig. 3, the downlink scheduler allocates resources to users based on their channel quality, QoS needs and fairness. eNodeB sends date and scheduling signaling based on scheduling results. Scheduled users accept data based on BR assignment results, MCS options and antenna selection in downlink scheduling signalling [14].

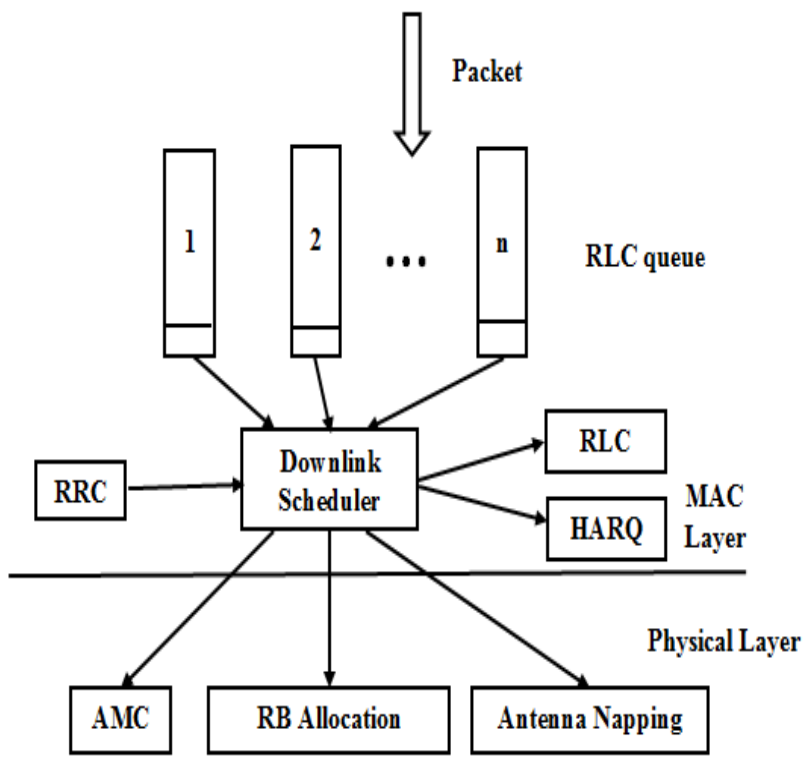

Figure.2 Position of scheduler in LTE

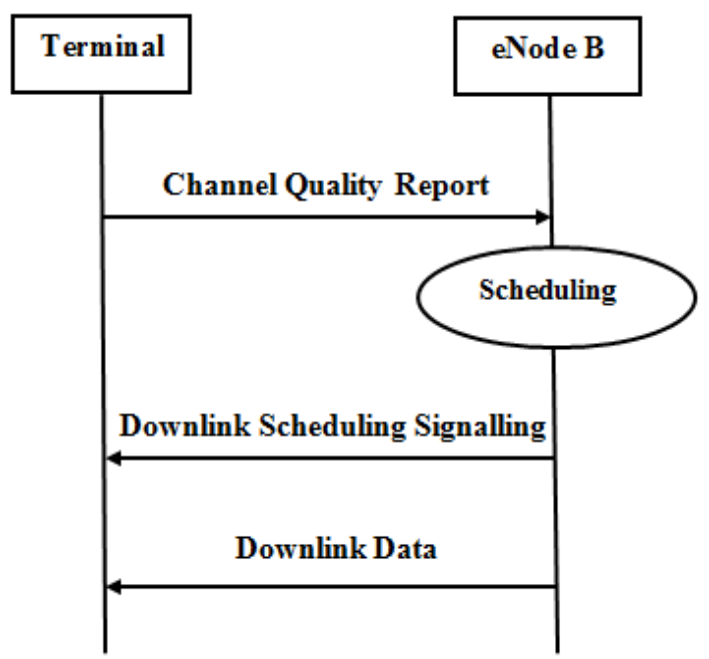

Figure.3 Downlink Scheduling Process

\subsection{Resource Allocation for M2M Communications}

The classifications of LTE / LTE-A scheduling techniques are based on M2M communication requirements to achieve optimal integration. Based on these characteristics, we can conclude that M2M communications require four main requirements. First, energy-efficient scheduling uses SC-FDMA for the uplink and OFDMA for the downlink. Second, QoS-based planning is required to handle different QoS requirements such as latency, jitter, error rate, and Guaranteed Bit Rate (GBR). Third, multi-hop based scheduling uses several hops to send data over short distances instead of sending them over long distances using a single hop, which saves energy. Finally, scalable network based scheduling uses the native IP LTE-A connectivity 
feature to support a considerable number of M2M communications [15].

Scheduling is the process implemented by eNodeB to allocate radio resource PRB to UE or MTCD in the downlink or uplink direction. The PRB is a resource grid consisting of 12 subcarriers within a time interval. The scheduling process can be divided into two stages:

Time Packet Packing Scheduling (TDPS): In this step, the eNodeB selects a terminal (UE or MTCD) or a group of terminals to which PRBs must be allocated according to certain criteria, for example: channel status, QoS and equity.

Frequency Domain Packet Scheduling (FDPS): In this step, the eNodeB selects the PRBs to assign to the terminal or group of terminals that were selected in the TDPS step. The eNB allocates PRBs that the terminal can use to the maximum. For example, it can allocate the PRBs for which the given terminal has the best channel conditions [16].

The design of downlink scheduling techniques for M2M communications should take into account the fact that MTCDs in M2M communications may have strict delay requirements. In addition, scheduling should take into account the fact that a significant number of MTCDs can contend for limited radio resources [17].

\section{Downlink resource scheduling algorithms}

Packet Scheduling mechanisms play a fundamental role in the radio resource management (RRM) in cellular networks as they aim to optimize, according to specific criteria, access to time and frequency resources. These mechanisms allow the allocation of radio resources by taking into consideration certain parameters relating to the state of the transmission channel and to the users' requirements in terms of QoS, especially for realtime streams, namely the voice and the video streams. The purpose of radio resource allocation algorithms is to improve system performance by increasing spectral efficiency and network equity [18]. It is therefore essential to find a compromise between efficiency (increase in throughput) and equity between users. The main purpose of this type of algorithm is to maximize the overall system throughput. Several algorithms use this approach as: Proportional Fair (PF), Maximum Largest Weight Delay First (MLWDF), Exponential Proportional Fair (EXP/PF), Frame Level Scheduler (FLS), Exponential (EXP) RULE and Logarithmic (LOG) RULE [19].

\subsection{Proportional fair}

It is an opportunistic scheduling algorithm. This type of algorithm uses infinite queues; these queues are used in the case of non-real time traffic. Its purpose is to try to maximize the overall throughput of the system by increasing the throughput of each user at the same time; it tries to ensure equity between users, the objective function representing the $\mathrm{PF}$ algorithm is:

$$
a=\frac{d_{i}(t)}{d_{i}}
$$

Where $d_{i}(t)$ is the rate corresponding to the CQI of the user $i$ and $d_{i}$ is the maximum rate supported by the Resource Blocks (RB).

\subsection{Exponential proportional fair}

This is an improvement of the PF algorithm that supports real-time flows (multimedia); by the way, it prioritizes real time flows over others. A user $\mathrm{k}$ is designated for the scheduling according to the following relation:

$$
\begin{gathered}
k=\max _{i} a_{i} \frac{d_{i}(t)}{d_{i}} \exp \left(\frac{a_{i} W_{i}(t)-X}{1+\sqrt{X}}\right) \\
X=\frac{1}{N} \sum_{i=1}^{N} a_{i} W_{i}(t)
\end{gathered}
$$

Where $W_{i}(t)$ is the time tolerated by the flow and $a_{i}$ is strictly positive setting for all $i$.

\subsection{Maximum largest weighted delay first}

M-LWDF is one of the algorithms considering time limits. This type of algorithm deals with delays in arriving and delivering packets, designed primarily to handle real-time flows (multimedia and voip). If a packet exceeds these tolerated delay values, it will be removed from the list of flows to schedule which significantly degrades the QoS. This algorithm supports flows with different QoS requirements; it tries to weight packet delays using channel state knowledge, at a time $t$, the algorithm chooses a user $\mathrm{k}$ for scheduling via the formula:

$$
k=\max _{i} a_{i} \frac{d_{i}(t)}{d_{i}} W_{i}(t)
$$

This is practically the same formula of the EXP-PF algorithm, except that: 


$$
a_{i}=-\log \left(p_{i}\right) T_{i}
$$

Where $p_{i}$ is the probability that the delay is not respected and $T_{i}$ is the time that the user $\mathrm{i}$ can tolerate.

This algorithm is mainly aimed at the real-time flow which requires the respect of the deadlines, it gives good results in this context, by cons for nonreal time flows, and it is really not a good choice because the delay is really not an important parameter.

\subsection{Frame level scheduler}

FLS is a multi-class scheduling algorithm; this scheduler class considers classes of queued flows in order to execute the appropriate scheduling policy for each class. The type of flow (whether real time or non-real time) is a fundamental parameter for this type of algorithm. Before making the resource allocation decision, the service type must be inspected before allocating the appropriate resource blocks for the broadcast. On the other hand, in spite of the prioritization of the real-time flows, the classical flows will not be neglected and removed from the queue in the event of congestion. FLS is a scheduler that considers quality of service and is mainly used for real-time communications in LTE networks. Its scheduling scheme is divided into two levels, which interact with each other to allow the dynamic allocation of resource blocks to users. In the upper level, a less complex algorithm based on a linear control loop and discrete over time; this level specifies the number of bits contained in the packet to be transmitted by the source in the LTE frame. In the lower level, the radio resources are allocated to the users using the scheduling strategy PF taking into account the bandwidth required by the FLS scheduler. This two-level modeling provides a compromise between system throughput and equity. For the scheduler PF the volume of the data $u_{i}(k)$ for the i flux in the $\mathrm{k}$ LTE frame is calculated according to the following formula:

$$
u_{i}(k)=h_{i}(k) * q_{i}(k)
$$

In the preceding formula, the volume of data to be transmitted is the convolution in the discrete time (*) between the level of the queue $q_{i}(k)$ and the impulse response of the linear filter used denoted $h_{i}(k)$.

\subsection{Exponential RULE}

The exponential scheduler is considered an enhancement of the EXP/PF scheduler; it uses at most the parameters used in EXP/PF: the spectral efficiency of the user is one of the optimization parameters that are set by report to the need of the network. The EXP-RULE provides quality of service guarantees over a wireless link. EXP-RULE selects a single user per queue to receive the service in each scheduling instant. EXP-RULE is based on the following expression:

$$
\begin{aligned}
& w_{i, k}{ }^{\text {EXPrule }} \\
& =b_{i} \exp \left(\frac{\alpha_{i} D_{H O L, i}}{c+\sqrt{\left(\frac{1}{N_{r t}}\right) \sum_{i} D_{H O L, i}}}\right) \cdot \Gamma_{k}^{i}
\end{aligned}
$$

Where $N_{r t}$ represents the number of active real time flows, $D_{H O L, i}$ represents the timeout of the packet in the queue and $\Gamma_{k}^{i}$ is the spectral efficiency of the user $\mathrm{i}$ for the flow $\mathrm{k}$. For optimal results, the parameters $\alpha \mathrm{i}$, bi and $\mathrm{c}$ are defined as follows:

$$
\left\{\begin{array}{c}
\alpha_{i} \in\left[\frac{5}{\left(0.99 \tau_{i}\right)}, \frac{10}{\left(0.99 \tau_{i}\right)}\right] \\
b_{i}=1 / E\left[\Gamma^{i}\right] \\
c=1
\end{array}\right.
$$

It should be noted that the EXP-RULE scheduler takes into account the delay taken by a user's packet and normalize it with respect to the overall delay experienced by the UEs of the cell. The advantage of this scheduler is that it relies on the state of the network (here the LTE cell) before approaching the scheduling process.

\subsection{Logarithmic RULE}

LOG-RULE is a scheduler that ensures a balance in the quality of service parameters in terms of average delay. This algorithm is based on the same parameters used in the EXP-RULE scheduler; however, the scheduler metric is computed from the logarithm of the user's flow. The LOG-RULE is represented as follows:

$$
w_{i, k}^{\text {LOGrule }}=b_{i} \log \left(c+\alpha_{i} D_{H O L, i}\right) \cdot \Gamma_{k}^{i}
$$

Where $D_{H O L, i}$ represents the timeout of the packet in the queue, and $\Gamma_{k}^{i}$ is the spectral efficiency of the user $\mathrm{i}$ for the flow $\mathrm{k}$. 
The fairness and optimal bit rate of the LTE cell are obtained by choosing the right optimization parameters $\alpha \mathrm{i}$, bi and $\mathrm{c}$. For optimal results, the parameters $\alpha \mathrm{i}$, bi and $\mathrm{c}$ are defined as follows :

$$
\left\{\begin{aligned}
\alpha_{i} & \in \frac{5}{\left(0.99 \tau_{i}\right)} \\
b_{i} & =1 / E\left[\Gamma^{i}\right] \\
c & =1.1
\end{aligned}\right.
$$

\section{Proposed method}

In this section, we propose a resource scheduling method for the downlink in LTE for M2M communications. This method provides a QoS guarantee for real-time services and is also suitable for non-real-time services. Different services have QoS requirements in the LTE system; on this basis 3GPP divides them into several types. Given division, we prioritize different types of services to ensure real-time service delivery with high QoS requirements. At the same time, we take into account the priority of the logical channel of the Media Access Control (MAC) layer and consider the user's channel quality to obtain a higher cell rate.

Existing scheduling algorithms including PF, MLWDF, EXP PF, FLS, EXP-RULE and LOGRULE have a common disadvantage. None of them takes into account the QoS needs of the user's services. Sometimes there are a lot of users in the system and non-real-time services need too many resources, in which case this type of algorithm cannot guarantee real-time service performance. There are certain algorithms considering the quality of service of the user for the OFDMA system. One is $\mathrm{PF}$, the user with a maximum packet transmission delay at the highest priority, and this algorithm gives high results in terms of delay and packet loss rates. Considering that it can neither guarantee the flow of the system, nor be suitable for real-time services. Therefore, according to the limitation of the method above, we propose a method of scheduling algorithm. It combines QoS priority with MAC service and logical channel prioritization with user channel quality indication and user equity. It applies not only to real-time services but also to non-realtime services.

The resource allocation method that we will describe performs the scheduling function of Fig. 3. In the proposed method, packet scheduling executes resource allocation decisions every $1 \mathrm{~ms}$ which is defined as the Transmission Time Interval (TTI), for real-time service is certainly prior to the non-realtime service and the allocation of resources for the service in real time is prioritized at non real time.
We consider in this method of resource allocation, $\mathrm{RB}_{\text {video }}$ is the number of resources allocated for video traffic with $\mathrm{RB}_{\text {video_max }}$ is maximum number of resources that can be assigned to users who uses video traffic, $\mathrm{RB}_{\text {voip }}$ is the number of resources allocated for voip traffic with $\mathrm{RB}_{\text {voip_max }}$ is the maximum number of resources that can use voip traffic and RBnon_real_time these are the resources allocated for non real-time traffic with RBnon_real_time_max is the maximum number of resources that can use non-real-time traffic. The diagram of the proposed process is illustrated in Fig. 4.

We will present our resource scheduling algorithm in two steps.

Resource allocation for real-time services: If there are real-time services, we allocate resources to them preferentially. If all available real-time resources are exhausted, then serve the non-realtime services in turn. When allocating resources for real-time services, we must take into account the quality of user channels, the delay of packet transmission and the prioritization of logical channels.

Resources allocation for non-real-time services: We are starting to allocate resources for non-realtime services after all real-time services have been served or the real-time available resource is exhausted. Given their low demand for QoS and to reduce complexity, we refer to delay and loss rate factors. If all non real-time services have been served before the non-real-time resource is exhausted, the rest is real-time.

\section{Results and discussion}

In this section, we evaluate the performance of our proposed method by applying it on the existing LTE downlink schedulers which are PF, MLWDF, EXP/PF, FLS, EXP-RULE and LOG-RULE, already studied in section 3 .

According to this existing works [20-22] concentrated on LTE scheduling downlink algorithms, the authors compared and analyzed LTE downlink schedulers applied at the level of specific scenarios and separate traffics either in real time or in non real time, therefore, this is not applicable in an environment with multiple services for heterogeneous users. So, the objective of our work is to propose a new method for M2M communications, which meets this requirement by applying it on LTE downlink schedulers in order to conclude which is the most efficient and performing algorithm which achieve high spectral efficiency in cellular systems, maximizing throughput and provide quality of 
service guarantees for system users, by making a differentiation between the traffics and especially we give more priority to real time traffic than non real time traffic. Video and voip were selected as real-time traffic and Best Effort (BE) for non-real time.

We implemented our new scheme in a M2M scenario based on the priority and differentiation between heterogeneous traffic using an open source LTE-Sim simulator. LTE-Sim is a LTE network event simulator, developed in $\mathrm{C}++$ language. In addition, it supports multiple scenarios, single and multi-cell environments, QoS management, user mobility, transfer and it was designed to perform simulations for different uplink and downlink scheduling strategies [23].

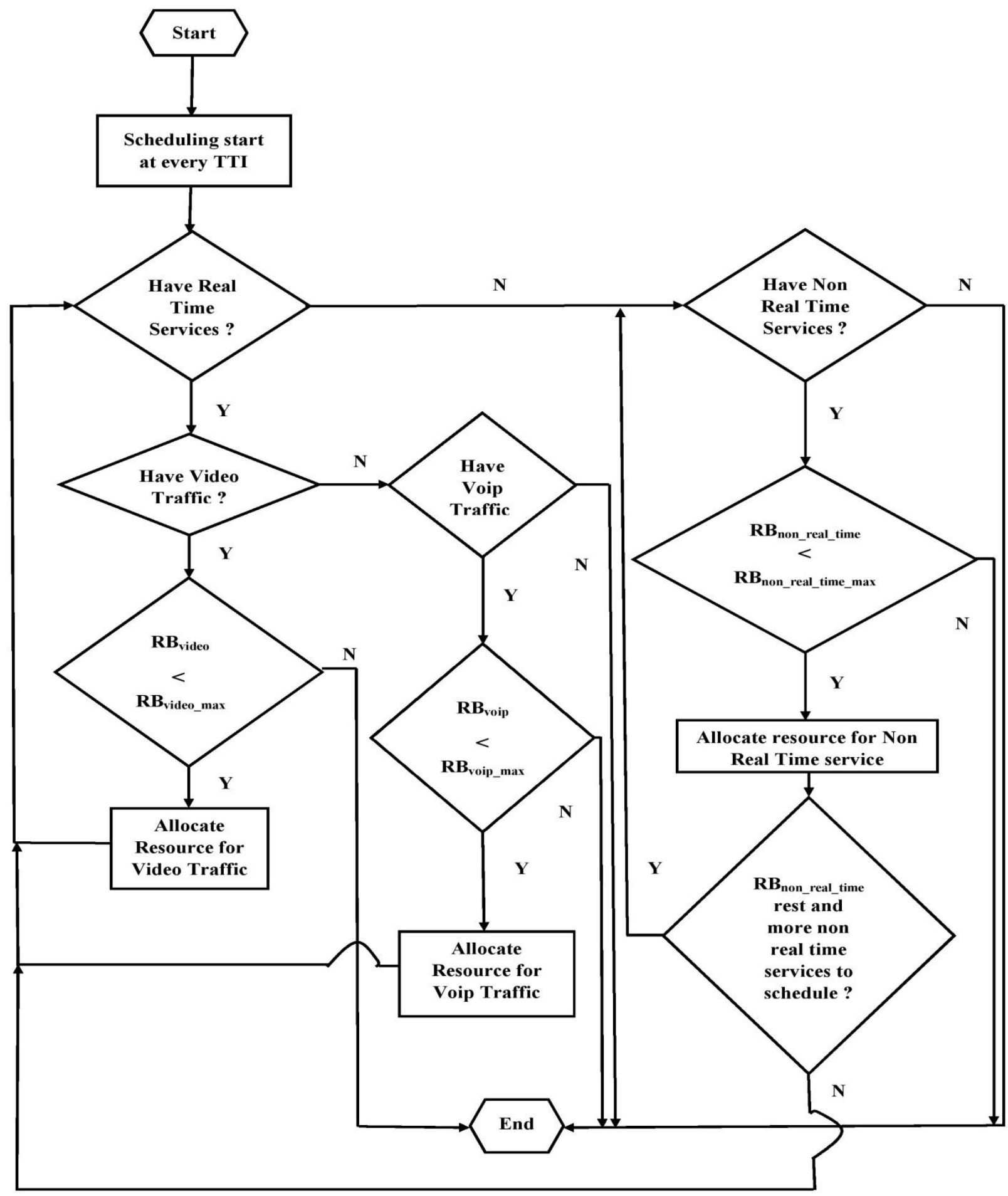

Figure.4 Diagram of the proposed method 
Table 1. Simulation Parameters

\begin{tabular}{|c|c|}
\hline Simulation Parameters & Values \\
\hline Simulation duration & $120 \mathrm{~s}$ \\
\hline Flow duration & $120 \mathrm{~s}$ \\
\hline Frame structure & FDD \\
\hline Mobility & $1 \mathrm{Km}$ \\
\hline Cell radius & $20 \mathrm{MHz}$ \\
\hline System Bandwidth & $180 \mathrm{kHz}$ \\
\hline RB Bandwidth & $0.5 \mathrm{~ms}$ \\
\hline Time Slot & $1 \mathrm{~ms}$ \\
\hline Scheduling Time (TTI) & $100 \mathrm{RBs}$ \\
\hline Number of RBs & $0.1 \mathrm{~s}$ \\
\hline Maximum delay & $242 \mathrm{kbps}$ \\
\hline Video Bit Rates & $8.4 \mathrm{kbps}$ \\
\hline VoIP Bit Rates & 0 à 100 \\
\hline Number of MTCDs & \\
\hline
\end{tabular}

In our simulation, we considered the case of a single cell with interference; we used an environment of a cell with a radius of 1 kilometer in which a set of MTC users set chosen in the range [0100] and uniformly in random direction mobility. The purpose of this simulation is to evaluate the performance of the LTE network in high congestion M2M scenario based on the method of priority and differentiation between real time and non-real time traffics proposed in this paper, users are uniformly distributed and for every two users transmitting video traffic and two users transmitting voip traffic there is one user transmitting BE traffic. Our evaluation is based on schedulers implemented in the base stations of the LTE network which are PF, MLWDF, EXP/PF, FLS, EXP-RULE and LOGRULE by the measurement of Throughput, Goodput, Fairness, PLR, Delay and Spectral Efficiency [24, $25]$. The simulation parameters are illustrated in the following table.

The delay has a very important influence on the performance of the network. Latency is the transit time of a packet from eNodeB to the device. It depends on the nature of the applications present in a network that the degree of requirement changes likes propagation time, processing time and packet size. As can be seen in Fig. 5, for the scheduling algorithm PF, the delay is in high increments until becoming intolerable with a significant timeout value because the PF algorithm satisfies the service scheduling requirements not real time, but is not ideal for real-time services. This is because the PF

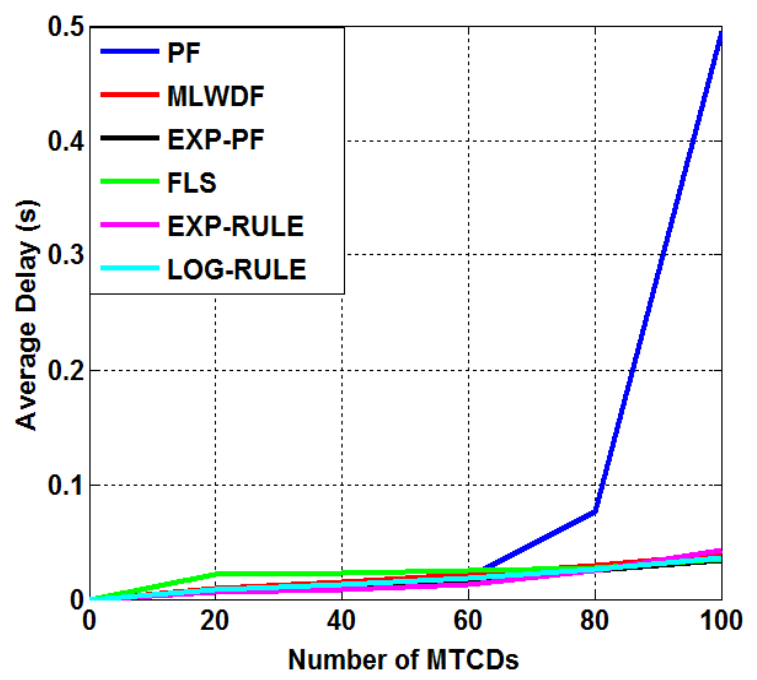

Figure.5 Average delay against number of MTCDs

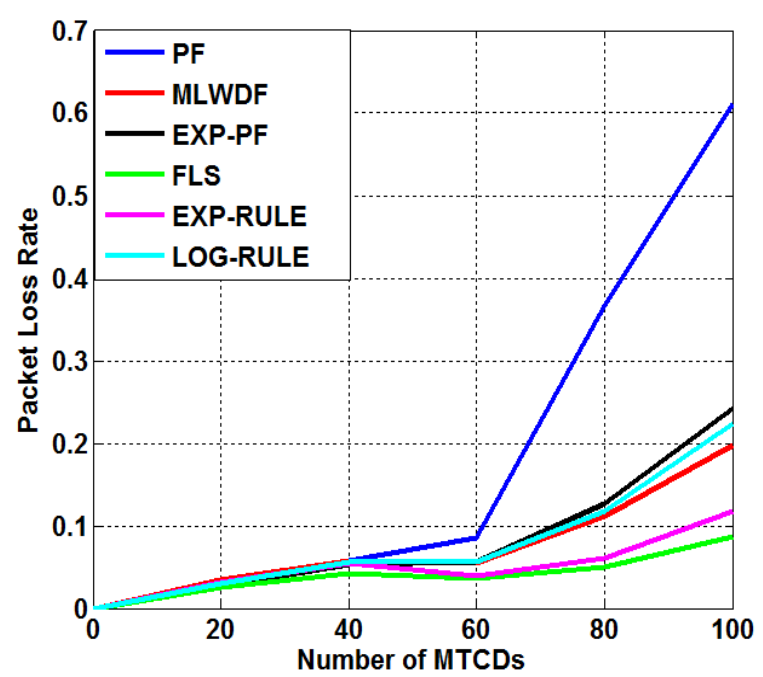

Figure.6 Packet Loss Rate against number of MTCDs

scheme did not take into account the delay of the data packet which is one of the attributes of the LTE network. For the other algorithms studied, the delay remains the weakest and is negligible and insensitive to the increase of devices in the cell.

The PLR Estimation improvement is a critical issue because its value has a big effect on network performance, especially when it comes to real-time traffic like voice over IP and video. Packet loss is the lost bytes when transmitting packets. It expresses itself in rate of loss. Rather, it corresponds to the number of data packets that were not received by the destination during a communication. This can be caused by many factors, mostly due to network congestion, as it can be caused by packet latency.

Fig. 6 represents the evolution of the packet lost rate as a function of the number of MTCDs in the coverage area of the cell, when the number of active devices increases in the cell, the loss rate increases, 




Figure.7 Average throughput against number of MTCDs

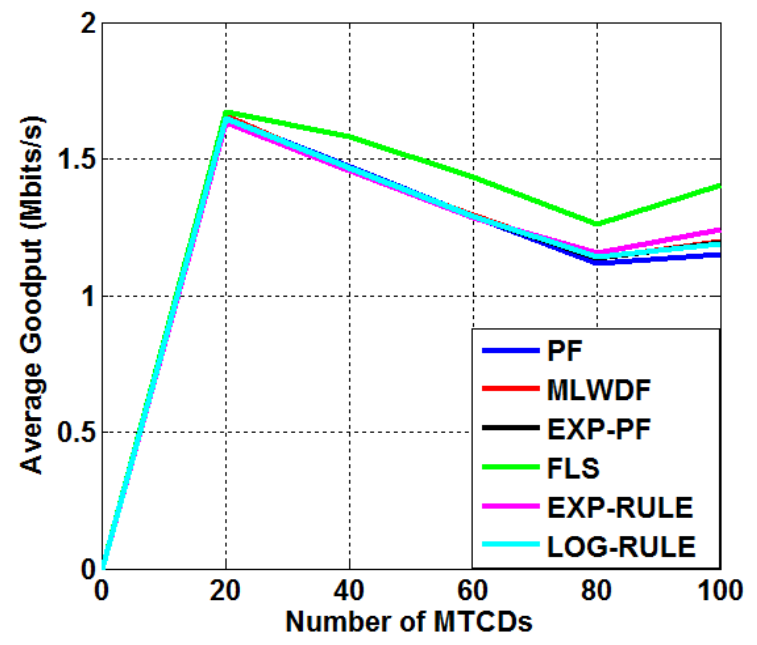

Figure.8 Average goodput against number of MTCDs

in particular for the PF algorithm which could reach a maximum rate of $60 \%$.

Fig. 7 illustrates the average throughput as a function of numbers of MTC devices, the measurement of this metric is one of the important operations which makes it possible to identify the average success rate of transfer of the messages on a communication channel, its value is calculated for a time interval, by dividing the total amount of information received during this interval by the duration of the interval in question. As shown in Fig. 7, the FLS algorithm offers the largest throughput for the video stream case, followed by the EXPRULE. The EXP-PF, MLWDF and LOG-RULE algorithms are very comparable in terms of throughput performance that starts to decrease from 80 devices. For the PF algorithm, its bit rate also decreases in the same direction as the other algorithms but from 40 devices and its value is much lower this is explained by, when the number of MTC devices is increased, the delay (Fig. 5) and packet loss rate (Fig. 6) increase. The decrease in the throughput is due to the unavailability of block resources sufficient to serve all users.

Goodput is the average rate of a successful transmission of data over a communication channel. In our simulation, this measure measures only the total data rate on the network, ignoring all other headers. A user's goodput is measured by first counting the total number of successfully received data packets and calculating the number of bits received, which is ultimately divided by the total execution time of the simulation. Fig. 8 shows that the FLS algorithm offers better performance in terms of goodput compared to other algorithms that have almost the same values where the goodput decreases according to the number of MTC devices in the cell. We also note that the PF is the least efficient with regard to the metric PF.

Fig. 9 shows the calculated fairness index for our scenario which is obtained by considering the throughput achieved by each flow at the end of each simulation, it is noted here that the fairness rates through the EXP-PF, MLWDF, FLS EXP-RULE and LOG-RULE are almost similar and believe in the density of the cell, reaching almost $90 \%$ equity between different users. On the other hand, the PF decreases from 60 MTCDs.

Spectral efficiency is defined as the maximum user rate divided by the bandwidth of the channel; this is the number of received bits correctly normalized by the resource consumed in time and bandwidth. Thus, spectral efficiency is strongly related to resource consumption and packet error rate. As shown in Fig. 10, the MLWDF algorithm has a better spectral efficiency compared to that measured for the other algorithms. This efficiency decreases for all the algorithms as the number of devices increases.

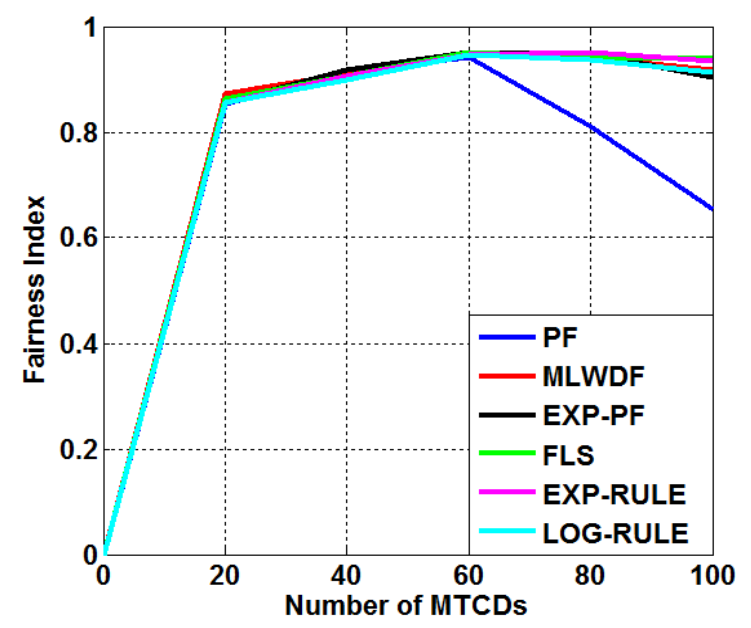

Figure.9 Fairness index against number of MTCDs 


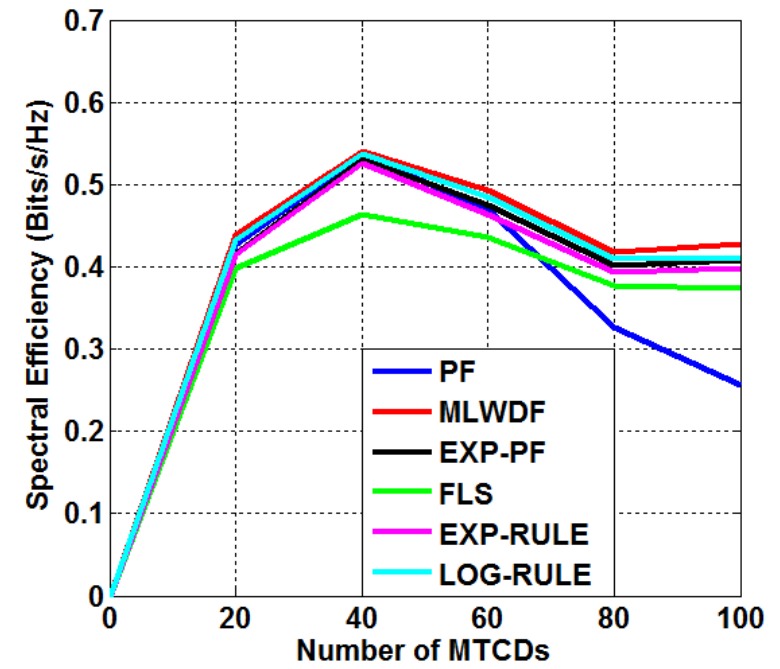

Figure.10 Spectral efficiency against number of MTCDs

\section{Conclusion}

In this paper, a new resource scheduling method based on the priority and differentiation of services for M2M communications in LTE downlink networks has been proposed. We consider two types of traffic: real-time traffic (video and voip) and nonreal-time traffic (best effort), we assign RBs to MTC devices that use real-time service ahead of non-real time to satisfy service QoS in real time. We presented a comparative analysis of six radio resource allocation algorithms based on the proposed method, in order to establish the impact of these resource allocation algorithms on the quality of service of different applications for a M2M scenario in LTE networks. Simulations were made in terms of throughput, goodput, delay, fairness, packet loss rate and spectral efficiency to conclude the most efficient algorithm. We can notice that the FLS downlink scheduler based on the proposed priority method in our simulation has better performance due to their characteristics of less delay and PLR, high throughput, goodput and fairness. We can conclude that the FLS algorithm is the suitable scheduler in a hybrid environment with heterogeneous traffic and where we prioritize the real time traffic that non real time traffic. For future work the performance of different scheduling algorithms on LTE network for M2M communications can be analysed under different applications (CBR, VBR, UBR and ABR).

\section{References}

[1] J. Kim, J. Lee, J. Kim, and J. Yun, "M2M service platforms: Survey, issues, and enabling technologies", IEEE Communications Surveys Tutorials, Vol.16, No.1, pp.61-76, 2014.
[2] T. Taleb and A. Kunz, "Machine type communications in 3GPP networks: Potential, challenges and solutions", Communications Magazine, Vol.50, No.3, pp.178-184, 2012.

[3] M. Ouaissa, M. Benmoussa, A. Rhattoy, M. Lahmer, and I. Chana, "Performance Analysis of Random Access Mechanisms for Machine Type Communications in LTE Networks", In : Proceedings of the Int. Conf. on Advanced Communication Systems and Information Security 2016 (ACOSIS'16), Marrakech, Morocco, 2016.

[4] M. Ouaissa, M. Benmoussa, A. Rhattoy, M. Lahmer, and I. Chana, "Impact of M2M Traffic in Random Access Channel over LTE Networks", In: Proc. of the lst Springer International Conference on Emerging Trends and Advances in Electrical Engineering and Renewable Energy, India, 2016.

[5] A. Biral, M. Centenaro, A. Zanellan, L. Vangelista, and M. Zorzi, "The challenges of M2M massive access in wireless cellular networks", Digital Communications and Networks, Vol. 1, No. 1, pp.1-19, 2015.

[6] 3GPP TS 22.368. V14.0.1, "Service requirements for Machine-Type Communications (MTC); Stage 1 (Release 14)", 2017-08.

[7] A. Aijaz, and A. Hamid Aghvami, "On Radio Resource Allocation in LTE Networks with Machine-to-Machine Communications", In: Proc. of the 2013 IEEE 77th Vehicular Technology Conference (VTC Spring), Dresden, Germany, 2014.

[8] F. Ghavimi and H. H. Chen, "M2M Communications in 3GPP LTE/LTE-A Networks: Architectures, Service Requirements, Challenges, and Applications", IEEE Communications Surveys Tutorials, Vol. 17, No. 2, 2015.

[9] G. Fritze, SAE: The Core Network for LTE, 2012.

[10] S. Sesia, I. Toufik, and M. Baker, LTE-The UMTS Long Term Evolution: From Theory to Practice, 2nd edition, 2011.

[11] M. Coupechoux and P. Martins, Vers les systèmes radio mobiles de 4 e génération - de l'UMTS au LTE, 2013.

[12] 3GPP TS 36.300. V15.2.0, "Evolved Universal Terrestrial Radio Access (E-UTRA) and Evolved Universal Terrestrial Radio Access Network (E-UTRAN); Overall description; Stage 2 (Release 15)”, 2018. 
[13] 3GPP TS 36.211. V8.0.0, "Evolved Universal Terrestrial Radio Access (E-UTRA); Physical Channels and Modulation," 2018-06.

[14] 3GPP TS 36.321. V15.2.0, "Evolved Universal Terrestrial Radio Access (E-UTRA); Medium Access Control (MAC) protocol specification Release 15)", 2018-07.

[15] T. P. C. de Andrade Tiago, A. A. Carlos, and L. S. da Fonseca Nelson, "Allocation of Control Resources for Machine-to-Machine and Human-to-Human Communications Over LTE/LTE-A Networks", IEEE Internet of Things Journal, Vol. 3, No. 3, pp. 366-377, 2016.

[16] K. Zheng, F. Hu, W. Xiangy, M. Dohler, and W. Wang, "Radio Resource Allocation in LTE-A Cellular Networks with M2M Communications", IEEE Commun. Letters, Vol. 1, No. 3, pp. 209-212, 2012.

[17] R. Boisguene, S. C. Tsengy, C.W. Huang, and P. Lin, "A Survey on NB-IoT Downlink Scheduling: Issues and Potential Solutions", In: Proc. of 2017 13th International Wireless Communications and Mobile Computing Conference, 2017.

[18] Y. Bouguen, E., and Hardouin, F.X. Wolff, LTE et les réseaux 4G, 2012.

[19] S.B. Monikandan, A. Sivasubramanian, and S.P.K. Babu, "A Review of MAC Scheduling Algorithms in LTE System", International Journal on Advanced Science, Engineering and Information Technology, Vol. 7, No. 3, pp. 1056-1068, 2017.

[20] S.S. Fouziya and R. Nakkeeran, "Study of Downlink Scheduling Algorithms in LTE Networks", Journal of Networks, Vol.9, No.12, 2014.

[21] A. Biernacki, and K. Tutschku, "Comparative Performance Study of LTE Downlink Schedulers", Wireless Pers Commun, 2013.

[22] S. Dardouri, and R. Bouallegue, "Comparative Study of Scheduling Algorithms for LTE Networks", World Academy of Science, Engineering and Technology International Journal of Computer, Electrical, Automation, Control and Information Engineering, Vol. 8, No. 3, 2014.

[23] G. Piro, L.A. Grieco, G. Boggia, F. Capozzi, and P. Camarda, "Simulating LTE Cellular Systems: an Open Source Framework", IEEE Transactions on Vehicular Technology, Vol.60, No.2, pp.498-513, 2010.

[24] A.M. Sahibzada, F. Khan, M. Ali, G.M. Khan, and Z.Y. Faqir, "Fairness Evaluation of Scheduling Algorithms for dense M2M
Implementations", In: Proc. of IEEE WCNC 2014 - Workshop on IoT Communications and Technologies, 2014.

[25] I.M. Delgado-Luque, F. Blánquez-Casado, M. Garcia Fuertes, G. Gomez, M.C. AguayoTorres, J.T. Entrambasaguas, and J. Baños, "Evaluation of Latency-Aware Scheduling Techniques for M2M Traffic Over LTE", In: Proc. of the 2012 Proceedings of the 20th European Signal Processing Conference, 2012. 